\title{
Trends of Antifungal Use Based on Sales Data in Japan from 2006 to 2015
}

\author{
Ayako Kawabe, ${ }^{a}$ Yuichi Muraki, ${ }^{* a}$ Ryo Inose,${ }^{a}$ Yoshiki Kusama,${ }^{b}$ Ryota Goto, ${ }^{a}$ Ai Ebisui, ${ }^{a}$ \\ Saki Ishii, ${ }^{a}$ Masahiro Ishikane, ${ }^{b}$ Hiroki Ohge, ${ }^{c}$ and Norio Ohmagari ${ }^{b}$ \\ ${ }^{a}$ Department of Clinical PharmacoEpidemiology, Kyoto Pharmaceutical University; 5 Misasaginakauchicho, \\ Yamashina-ku, Kyoto 607-8414, Japan: ${ }^{b}$ AMR Clinical Reference Center, Disease Control and Prevention Center, \\ National Center for Global Health and Medicine; 1-21-1 Toyama, Shinjuku-ku, Tokyo 162-8655, Japan: and \\ ${ }^{c}$ Department of Infectious Diseases, Hiroshima University Hospital; 1-2-3 Kasumi, Minami-ku, Hiroshima 734-8551, \\ Japan.
}

Received March 31, 2020; accepted May 29, 2020

The detailed epidemiology of invasive mycoses and superficial mycoses has not been clarified in Japan. In addition, treatment options have increased because of novel antifungals and/or guidelines for fungal infection. In the present study, we aimed to clarify the trends of antifungal use in Japan from 2006 to 2015 based on sales data to serve as an alternative indicator of fungal infection trends. We found that the total antifungal use decreased over time $\left(r=\mathbf{0 . 0 5 7}, P_{\text {for trend }}<\mathbf{0 . 0 0 0 1}\right)$. Oral and parenteral use significantly decreased by $44.1 \%\left(r=-0.056, P_{\text {for trend }}<0.0001\right)$ and $27.1 \%\left(r=-0.0012, P_{\text {for trend }}=0.00061\right)$, respectively. The trend of antifungal use for superficial mycoses significantly decreased by $49.8 \%\left(r=-0.061, P_{\text {for }}\right.$ trend $\left.<0.0001\right)$. However, the trend of antifungal use for invasive mycoses was significantly increased by $19.9 \%(r=0.0032$, $P_{\text {for trend }}=\mathbf{0 . 0 0 0 4 5}$ ). In Japan, the increase in the number of immunocompromised patients might be associated with the increase in the frequency of antifungal use for invasive mycoses. This is the first study to clarify the trends of antifungal use in Japan. Further research is needed to establish a continuous surveillance system to compare fungal infections between Japan and the world.

Key words antifungal use; invasive mycosis; superficial mycosis; surveillance

\section{INTRODUCTION}

Fungal infections are broadly classified as invasive and superficial mycoses. Invasive mycosis has a low morbidity rate and high mortality rate ${ }^{1,2)}$ and is one of the major complications in immunocompromised patients. The number of immunocompromised patients worldwide, including Japan, has increased owing to the increase in the population of elderly people $^{3,4)}$ and the incidence of cancer. ${ }^{5,6)}$ Although it is expected that the number of patients suffering from invasive mycosis will have further increased, the change in the incidence is unknown. In contrast, the exact incidence rate and trend for the incidence of superficial mycoses in Japan are not clarified. Therefore, the detailed epidemiology of invasive mycoses and superficial mycoses has not been clarified in Japan.

In recent years, the treatment options for invasive mycoses have increased. Antifungals such as echinocandin, voriconazole (VRCZ), liposomal amphotericin B (L-AMB), and posaconazole, which are indicated for invasive mycoses, have been released. In addition, topical drugs such as luliconazole and efinaconazole, which are indicated for superficial mycoses, have also been released. Furthermore, the guidelines for invasive mycoses ${ }^{7)}$ and superficial mycoses ${ }^{8)}$ have been published in Japan. Thus, it is considered that the treatment options are ever evolving. In Europe, because antifungal use is surveyed in each country every year, ${ }^{9)}$ it is possible to evaluate changes in the use of antifungals. However, the trends of antifungal use in Japan are still unclear.

In the present study, we aimed to clarify the trends of antifungal use in Japan from 2006 to 2015 based on sales data to serve as an alternative indicator of the trends of fungal infections.

\section{MATERIALS AND METHODS}

Data Collection Sales data were obtained from IQVIA Services Japan K.K. (Tokyo, Japan). The surveillance period was from 2006 to 2015. The data were collected using pharmaceutical sales distribution channels via wholesalers. The antifungal drugs were included in the Anatomical Therapeutic Chemical (ATC) code for systemic antifungal drugs (J02A). The data were analyzed according to the ATC classification by using a defined daily dose (DDD) as a measurement unit ${ }^{10)}$ (Table 1). DDDs are calculated by dividing the amount of drug used by the DDD. The 2019 version of the ATC/DDD system was applied to all data. The sales data were reported as DDDs

Table 1. DDD and Classification of Antifungal Drugs

\begin{tabular}{lccc}
\hline \hline \multirow{2}{*}{ Class } & Generic name & Oral & Parenteral \\
\cline { 3 - 4 } & & & DDD $(\mathrm{g})$ \\
\hline \multirow{2}{*}{ Polyene } & Amphotericin B & 0.4 & 0.035 \\
Azole & Liposomal amphotericin B & - & $0.15^{*}$ \\
& Itraconazole & 0.2 & 0.2 \\
& Voriconazole & 0.4 & 0.4 \\
\multirow{2}{*}{ Echinocandin } & Fluconazole & 0.2 & 0.2 \\
& Fosfluconazole & - & 0.2 \\
Others & Miconazole & - & 1 \\
& Micafungin & - & 0.1 \\
& Caspofungin & - & 0.05 \\
& Flucytosine & 10 & - \\
\hline
\end{tabular}

DDD; defined daily dose. *DDD was defined as 0.15 by using the dose of $2.5 \mathrm{mg} / \mathrm{kg}$ and the standard weight of $60 \mathrm{~kg}$. 
per 1000 inhabitants per day (DID), and the following equation was used:

DID (DDDs/1000 inhabitants/day)

Use of each year $(\mathrm{g})$

DDD $(\mathrm{g}) \times$ population of each year $(/ 1000$ inhabitants $) \times 365$ (days)

The DDD of L-AMB was not defined by WHO; thus, it was calculated as a standard weight of $60 \mathrm{~kg}$ for an adult dose of $2.5 \mathrm{mg} / \mathrm{kg}$ (described in the Ambisome ${ }^{\circledR}$ package insert) and was defined as $0.15 \mathrm{~g}$. The population data were obtained from the Statistics Bureau of Japan. ${ }^{11)}$

Antifungal drugs were assessed over time for each drug, dosage form (oral/parenteral), and purpose of use (superficial mycoses/invasive mycoses).

The purpose of use was classified into superficial mycoses and invasive mycoses on the basis of the indication in the package insert. Oral polyene (amphotericin B; AMPH-B), oral azole (itraconazole; ITCZ), allylamine (terbinafine; TERB), and other (nystatin; NYS) were defined as antifungals indicated for superficial mycoses. Oral azoles (fluconazole; FLCZ, VRCZ), parenteral azoles (ITCZ, FLCZ, fosfluconazole; F-FLCZ, VRCZ, miconazole; MCZ), parenteral polyenes (AMPH-B, L-AMB), parenteral echinocandins (micafungin; $\mathrm{MCFG}$, caspofungin; CPFG), and other (flucytosine; 5-FC) were defined as antifungals indicated for invasive mycoses.

Statistical Analysis and Ethical Consideration Time series analysis is generally performed in the evaluation of antibiotic use. Time series analysis requires more detail likely month and day etc. information to consider seasonal fluctuations. In addition, information on the intervention, which is the starting point of change, is also needed. However, this study used sales data on an annual basis and could not set the starting point of change. Thus, the trends of antifungal use were evaluated by performing linear regression. A value of $P_{\text {for trend }}<0.05$ was considered statistically significant for all analyses. The analyses were conducted using JMP ${ }^{\circledR} 14$ (SAS Institute Inc., Cary, NC, U.S.A.). Informed consent was waived because anonymized sales data were used.

\section{RESULTS}

Trends of Antifungal Use from 2006 to 2015 The investigated antifungals, which are classified as ATC code J02A systemic antifungals, included 12 components of 174 items. Figure 1 presents the trends of antifungal use from 2006 to 2015 . The total antifungal use significantly decreased by $43.4 \%$ from 2006 to $2015\left(r=-0.057, P_{\text {for trend }}<0.0001\right)$. Oral and parenteral use also significantly decreased by $44.1 \%$ $\left(r=-0.056, \quad P_{\text {for trend }}<0.0001\right) \quad$ and $27.1 \% \quad(r=-0.0012$, $\left.P_{\text {for trend }}=0.00061\right)$, respectively. Oral drugs accounted for $87.5 \%$ of the total antifungals.

The DID for oral drugs was high, and the drug with the highest DDD was TERB, followed by ITCZ, AMPH-B, FLCZ, and VRCZ. TERB accounted for more than $50 \%$ of antifungal use throughout the study period. However, the trend of TERB use decreased to $45.4 \%$ from 2006 to 2015 ( $\left.r=-0.047, P_{\text {for trend }}<0.0001\right)$. MCFG was the most used parenteral drug, and the DID had no significant change $\left(r=0.000058, \quad P_{\text {for trend }}=0.52\right)$. The DIDs of F-FLCZ, FLCZ, and VRCZ significantly decreased: $r=-0.0014$
$\left(P_{\text {for trend }}<0.0001\right), \quad r=-0.00017 \quad\left(P_{\text {for trend }}=0.00024\right)$, and $r=-0.00011\left(P_{\text {for trend }}<0.0001\right)$, respectively.

Trends of Antifungal Use Classified by Indication Figure 2 shows the trends of antifungal use classified by indication from 2006 to 2015. The trend of antifungal use for superficial mycoses significantly decreased by $49.8 \%$ from 2006 to $2015\left(r=-0.061, P_{\text {for trend }}<0.0001\right)$. However, the trend of antifungal use for invasive mycoses was significantly increased by $19.9 \%$ from 2006 to $2015\left(r=0.0032, P_{\text {for trend }}=0.00045\right)$.

\section{Trends of Antifungal Use}

Polyene

A significant decrease was observed for polyene use $\left(r=-0.0030, P_{\text {for trend }}=0.029\right)$. The trend of oral AMPH-B use significantly decreased $\left(r=-0.0031, P_{\text {for trend }}=0.028\right)$. From 2006 to 2008, the trend of parenteral AMPH-B use significantly decreased and then gradually decreased $(r=-0.00018$, $\left.P_{\text {for trend }}=0.0029\right)$. However, the trend of L-AMB use significantly increased $\left(r=0.00030, P_{\text {for trend }}=0.0035\right)$. For parenteral drugs, AMPH-B was frequently used until 2006, and the use of L-AMB has exceeded that of AMPH-B since 2007.

\section{Azole}

The total azole antifungal use was significantly decreased $\left(r=-0.0079, P_{\text {for trend }}<0.0001\right)$. For oral drugs, ITCZ accounted for most of the azole DID (52.7 to $71.3 \%$ ), followed by FLCZ and VRCZ. The trend of oral ITCZ use was significantly decreased $\left(r=-0.011, P_{\text {for trend }}<0.0001\right)$. However, the trends of oral VRCZ use $\left(r=0.0022, P_{\text {for trend }}<0.0001\right)$ and oral FLCZ use $\left(r=0.0022, P_{\text {for trend }}=0.00026\right)$ significantly increased. For parenteral drugs, the trends of VRCZ use $\left(r=-0.00011, P_{\text {for trend }}<0.0001\right)$ and FLCZ use $(r=-0.00017$, $P_{\text {for trend }}=0.00024$ ) significantly decreased.

Echinocandin

Echinocandin use as a whole significantly increased $\left(r=0.00048, P_{\text {for trend }}=0.00077\right)$. By contrast, no significant change was observed in the trend of MCFG use from 2006 to 2015 ( $\left.r=0.000058, P_{\text {for trend }}=0.52\right)$. CPFG was launched in 2012, and its use significantly increased from 2012 to 2015 $\left(r=0.00030, P_{\text {for trend }}=0.0010\right)$.

Other Antifungal Drugs

The trend of TERB use significantly decreased to $45.4 \%$ in 2015 compared with that in $2006(r=-0.047$, $\left.P_{\text {for trend }}<0.0001\right)$. There was no significant change in the trend of NYS use $\left(r=0.0000025, P_{\text {for trend }}=0.68\right)$, whereas the trend of 5-FC use significantly decreased $(r=-0.0000064$, $\left.P_{\text {for trend }}=0.0034\right)$.

\section{DISCUSSION}

This is the first study that clarifies the detailed trends of the antifungals used in Japan on the basis of sales volume. Total antifungal use was decreased over time, and the main reason for this result was the decrease in antifungal use for superficial mycoses. Interestingly, antifungal use for invasive mycoses increased.

Although oral TERB and ITCZ use accounted for more than half of total antifungal use in all periods, these drugs have significantly decreased to 45.4 and $58.5 \%$ from 2006 to 2015 , respectively. The main reason for the decrease in the total use of antifungals is the decrease in the use of oral TERB and ITCZ. The guidelines for dermatomycosis published in 2009 recommend not only oral but also topical 
(a) DID of total antifungals

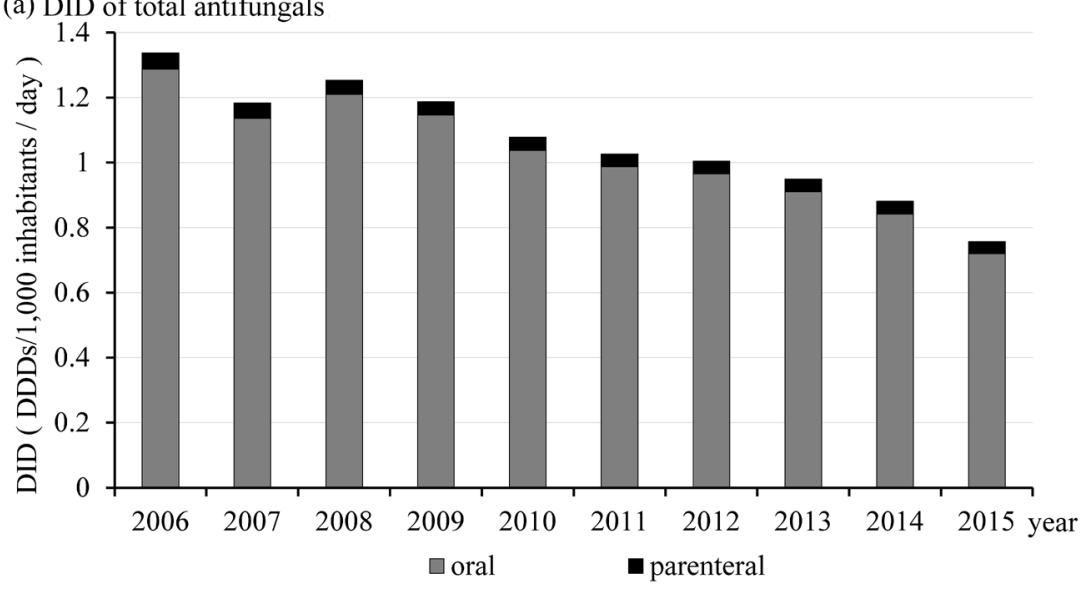

(b) DID of oral antifungals

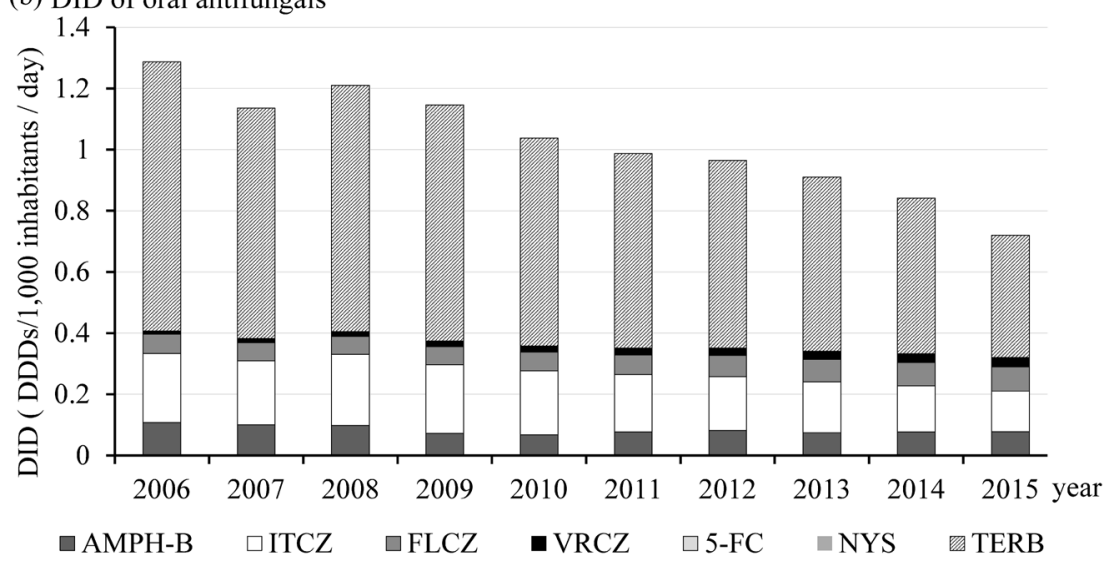

(c) DID of parenteral antifungals

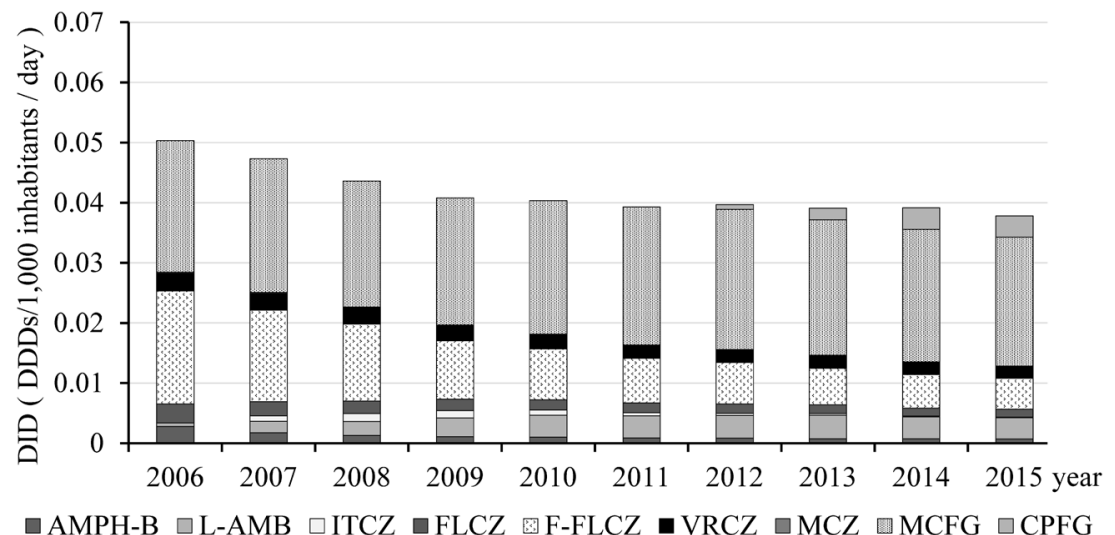

Fig. 1. Trends of Antifungal Use from 2006 to 2015

(a) DID of total antifungals, (b) DID of oral antifungals, and (c) DID of parenteral antifungals. AMPH-B, amphotericin B; ITCZ, itraconazole; FLCZ, fluconazole; F-FLCZ, fosfluconazole; VRCZ, voriconazole; 5-FC, flucytosine; NYS, nystatin; TERB, terbinafine; L-AMB, liposomal amphotericin B; MCZ, miconazole; MCFG, micafungin; $\mathrm{CPFG}$, caspofungin.

drugs. ${ }^{12)}$ It is possible that the launch of generic medicines caused a decrease in the use of oral TERB and ITCZ because the original medicine had been mainly decreased in this study (data not shown). Therefore, it is speculated that the decline in sales promotion of original medicine and the increasing use of topical drugs may have affected the decrease in the use of oral TERB and ITCZ.

The trend of antifungal use for invasive mycoses increased. It was considered that the increase in the number of immunocompromised hosts ${ }^{4,6)}$ in Japan is related to this finding.
Echinocandin accounted for more than half of the parenteral antifungals used for invasive mycoses. The use of echinocandin causes the FKS mutation of Candida glabrata, which leads to resistance to echinocandin. ${ }^{13)}$ Furthermore, the use of MCFG exerts a selective pressure toward resistant fungi such as Trichosporon species in medical institutions. ${ }^{14)}$ Even though the mechanism of antifungal resistance varies from that of antibacterial drugs, ${ }^{15)}$ the selective pressure of antifungals in a patient needs to be noted.

Oral azole use significantly increased, and parenteral azole 
(a) DID of total antifungals for superficial mycoses

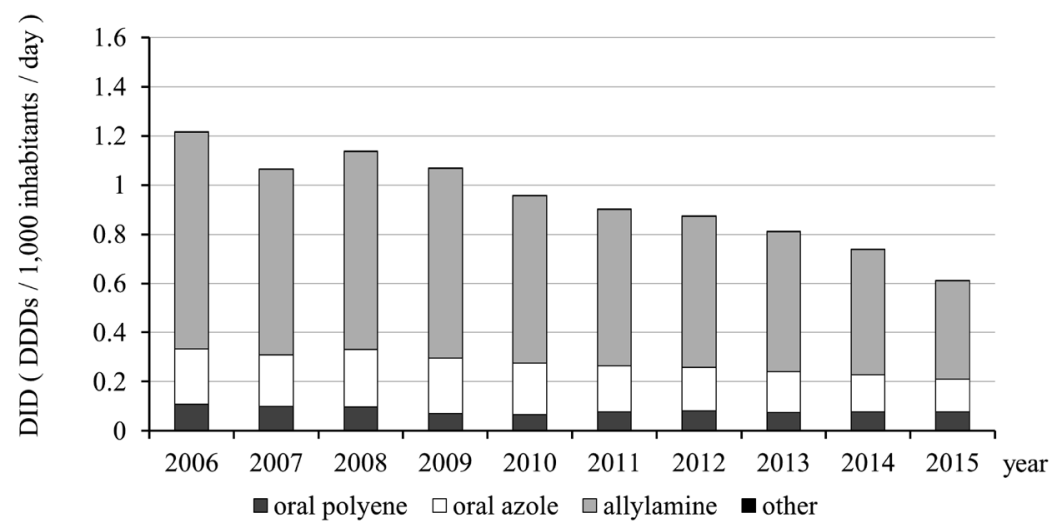

(b) DID of total antifungals for invasive mycoses

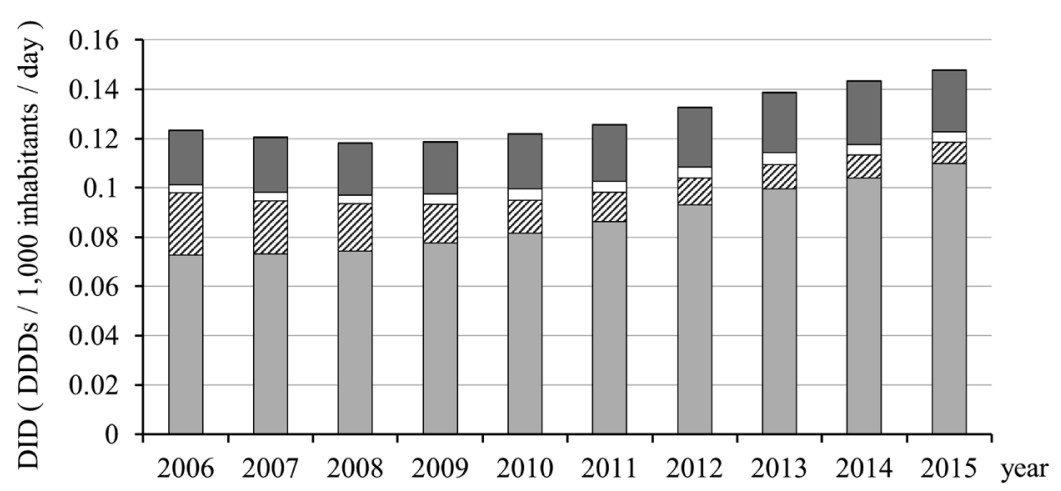

$\square$ oral azoles $\square$ pareneteral azoles $\square$ parenteral polyenes $\square$ parenteral echinocandins $\square$ other

Fig. 2. Trends of Antifungal Use Classified by Indication from 2006 to 2015

(a) DID of total antifungals for superficial mycoses and (b) DID of total antifungals for invasive mycoses. oral polyene: AMPH-B; oral azole: ITCZ; allylamine: TERB; other: NYS; oral azoles: FLCZ, VRCZ; parenteral azoles: ITCZ, FLCZ, F-FLCZ, VRCZ, MCZ; parenteral polyenes: AMPH-B, L-AMB; parenteral echinocandins: MCFG, CPFG; other: 5-FC. AMPH-B, amphotericin B; ITCZ, itraconazole; NYS, nystatin; TERB, terbinafine; FLCZ, fluconazole; VRCZ, voriconazole; 5-FC, flucytosine; L-AMB, liposomal amphotericin B; F-FLCZ: fosfluconazole; MCZ: miconazole; MCFG: micafungin; CPFG: caspofungin.

use significantly decreased. The Infectious Diseases Society of America published guidelines that recommended step-down treatment for oral azoles ${ }^{16)}$ and the prevention of invasive mycosis in patients with hematological malignancies. ${ }^{17)}$ In Japan, FLCZ and VRCZ obtained indications for the prevention of invasive mycosis in hematopoietic stem cell transplant patients in 2011 and 2015, respectively. As the number of patients with hematological malignancies in Japan is increasing, ${ }^{6)}$ it is speculated that oral azole prophylaxis for invasive mycoses has increased.

This study shows that the total antifungal use in Japan in 2007 was 1.06DID. However, a previous study reported that the range of antifungal use in Europe was from 0.45 to 3.03 DID. Antifungal use in Japan was comparable with that of Italy. ${ }^{18)}$ The present study was based on sales data, whereas the previous report was based on outpatient data. ${ }^{18)}$ Although it is difficult to make a direct comparison, the present study made it possible to compare the trends of antifungal use in Japan and Europe. There are few reports about the surveillance of antifungal use in the world. Therefore, it is necessary to establish a continuous surveillance system to make a comparison between Japan and the world regarding fungal infections.
This study has several limitations. First, given that sales volume was used in our study, the actual administered volume was not accurately reflected. Furthermore, the number of patients who used antifungals and the patients' background were unknown. Second, the most recent sales data were not included in this study. The information on antifungal use as old as possible needs to be evaluated in this study. Because the oldest available sales data was 2006, we purchased sales data from 2006 to 2015 for 10 years, based on funding. In the future, it will be necessary to continue the study with newer data and evaluate changes in antifungal use. Nevertheless, the results of this study are useful for understanding the overall exposure situation. This study also provides information on the promotion of the appropriate use of antifungals. In the future, it will be necessary to conduct continuous and detailed investigations on antifungal use by using other information, such as insurance claim data.

\section{CONCLUSION}

This is the first study to clarify the trends of antifungal use in Japan. Although the trend of total antifungal use decreased, the trend of antifungal use for invasive mycoses increased. 
Further research is needed to establish a continuous surveillance system to compare fungal infections between Japan and the world.

Acknowledgments This work was supported by the Ministry of Health, Labour and Welfare (Grant Number H31shinkougyousei-shitei-005, 19HA1004), JSPS KAKENHI Grant Number JP18K09957.

Conflict of Interest Yuichi Muraki received an honorarium for lecturing from Pfizer Japan, Inc. Other authors declare no conflict of interest.

\section{REFERENCES}

1) Cornely OA, Gachot B, Akan H, Bassetti M, Uzun O, Kibbler C, Marchetti O, de Burghgraeve P, Ramadan S, Pylkkanen L, Ameye L, Paesmans M, Donnelly JP. Epidemiology and outcome of fungemia in a cancer Cohort of the Infectious Diseases Group (IDG) of the European Organization for Research and Treatment of Cancer (EORTC 65031). Clin. Infect. Dis., 61, 324-331 (2015).

2) Lin SJ, Schranz J, Teutsch SM. Aspergillosis case-fatality rate: systematic review of the literature. Clin. Infect. Dis., 32, 358-366 (2001).

3) Permanyer I, Scholl N. Global trends in lifespan inequality: 1950-2015. PLOS ONE, 14, e0215742 (2019).

4) Statistics Bureau of Japan. "Elderly population.": «http://www.stat. go.jp/data/topics/topi1031.html, accessed 24 January, 2020.

5) Bray F, Ferlay J, Soerjomataram I, Siegel RL, Torre LA, Jemal A. Global cancer statistics 2018: GLOBOCAN estimates of incidence and mortality worldwide for 36 cancers in 185 countries. $C A$ Cancer J. Clin., 68, 394-424 (2018).

6) Cancer Information Service, National Cancer Center, Japan (Monitoring of Cancer Incidence in Japan (MCIJ)). "Cancer Registry and Statistics.”: 〈https://ganjoho.jp/reg_stat/statistics/dl/index.html〉, accessed 30 April, 2020.

7) Kohno S, Tamura K, Niki Y, et al. Executive summary of Japanese domestic guidelines for management of deep-seated mycosis 2014. Med. Mycol. J., 57, E117-E163 (2016).
8) Mochizuki T, Tsuboi R, Iozumi K, et al. Guideline for treatment of dermatomycosis 2019. Jpn. J. Dermatol, 129, 2639-2673 (2019).

9) European Centre for Disease Prevention and Control. "Annual reports and protocols on antimicrobial consumption.": 〈https://www. ecdc.europa.eu/en/antimicrobial-consumption/surveillance-anddisease-data/report-protocol>, accessed 10 February, 2020.

10) WHO Collaborating Centre for Drug Statics Methodology. “ATC/DDD index2019.”: 〈https://www.whocc.no/atc_ddd_index/〉, accessed 6 February, 2019.

11) Statistics Bureau of Japan. "Population estimate.”: 〈http://www.stat. go.jp/data/jinsui/2016np/index.htm>, accessed 6 February, 2019.

12) Watanabe $S$, Mochizuki $T$, Iozumi $K$, Katoh $T$, Sei $Y$, Mutoh $M$, Naka W, Nishimoto K, Hiruma M, Matsuda T. Guidelines for diagnosis and treatment of dermatophytosis. Jpn. J. Dermatol., 119, 851-862 (2009)

13) Pham CD, Iqbal N, Bolden CB, Kuykendall RJ, Harrison LH, Farley MM, Schaffner W, Beldavs ZG, Chiller TM, Park BJ, Cleveland AA, Lockhart SR. Role of FKS Mutations in Candida glabrata: MIC values, echinocandin resistance, and multidrug resistance. Antimicrob. Agents Chemother., 58, 4690-4696 (2014).

14) Matsue K, Uryu H, Koseki M, Asada N, Takeuchi M. Breakthrough Trichosporonosis in patients with hematologic malignancies receiving micafungin. Clin. Infect. Dis., 42, 753-757 (2006).

15) Cowen LE. Predicting the emergence of resistance to antifungal drugs. FEMS Microbiol. Lett., 204, 1-7 (2001).

16) Pappas PG, Kauffman CA, Andes D, Benjamin DK Jr, Calandra TF, Edwards JE Jr, Filler SG, Fisher JF, Kullberg BJ, Ostrosky-Zeichner L, Reboli AC, Rex JH, Walsh TJ, Sobel JD. Clinical practice guidelines for the management of candidiasis: 2009 update by the Infectious Diseases Society of America. Clin. Infect. Dis., 48, 503-535 (2009).

17) Freifeld AG, Bow EJ, Sepkowitz KA, Boeckh MJ, Ito JI, Mullen CA, Raad II, Rolston KV, Young JA, Wingard JR. Clinical practice guideline for the use of antimicrobial agents in neutropenic patients with cancer: 2010 update by the infectious diseases society of america. Clin. Infect. Dis., 52, e56-e93 (2011).

18) Adriaenssens N, Coenen S, Muller A, Vankerckhoven V, Goossens H. European Surveillance of Antimicrobial Consumption (ESAC): outpatient systemic antimycotic and antifungal use in Europe. $J$. Antimicrob. Chemother., 65, 769-774 (2010). 